\title{
Chemical and electrochemical characterization of Nafion containing silver nanoparticles in stripe-like distribution
}

\author{
B. Domènech ${ }^{a}$, V. Romero ${ }^{b}$, M.I. Vázquez ${ }^{b}$, M. Ávilac, J. Benavente ${ }^{b}$, M. Muñoz ${ }^{d}$ and J. Macanás*e
}

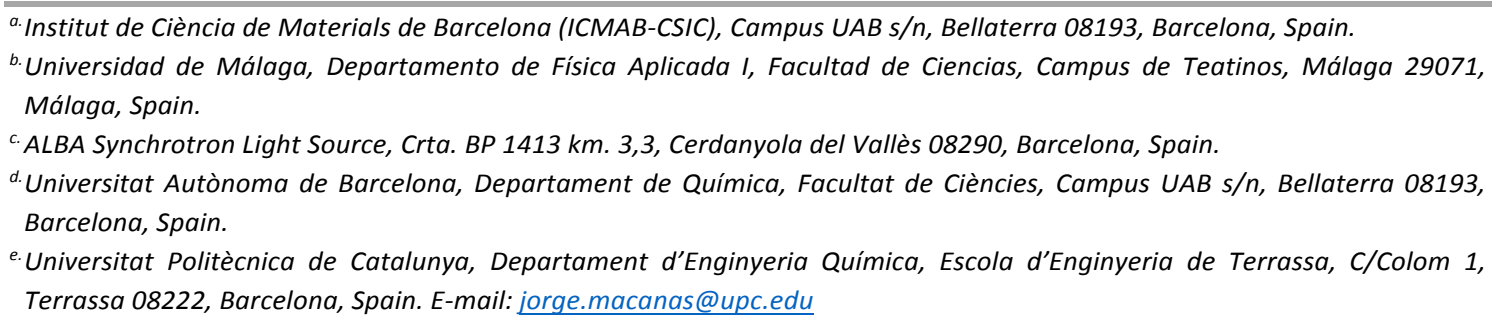

\section{Abstract}

The particular chemical structure of Nafion with nanostructured and segregated hydrophilic and hydrophobic domains enables its use as a model structure for the manufacture of polymer-metal nanocomposites. Among such nanocomposites, samples of Nafion-117 containing Ag-NPs of ca. $10 \mathrm{~nm}$ prepared by Intermatrix Synthesis exhibited a particular distribution in the form of stripes, a regular pattern that could reveal the real morphology of the polymer. To evaluate the potential application of this engineered material (e.g. in electrochemical devices), these new nanocomposite membranes were characterized by different techniques: microscopic, surface chemical analysis, mechanical and electrical/electrochemical. X-Ray (XRD and XPS) analyses combined with synchrotron experiments (XANES) were used to determine the chemical speciation of $\mathrm{Ag}$ in the membrane. Membrane potential and impedance spectroscopy measurements showed that such Ag-NPs did not hinder the diffusive transport of protons in the membrane bulk, moreover, they slightly reduced the electrical resistance in fully hydrated state samples. The mechanical evaluation of the nanocomposite evidenced the reduction of the elastic character when compared with the unmodified Nafion-117 sample.

\section{Introduction}

Nafion $^{1,2}$ is the most typical material for Proton Exchange Membranes (PEMs) ${ }^{3,4}$ and it is also a common choice for other electrochemical applications. ${ }^{5,6}$ That wide range of applications comes up due the exceptional properties of the polymer (i.e. high cation transport and chemical stability) which are determined, at last instance, by its chemical structure: a polytetrafluoroethylene (PTFE) backbone and regularly spaced long perfluorovinyl ether pendant side chains ended by sulfonic groups, which are responsible for the high ion exchange capacity. ${ }^{1}$

Regarding the morphology of Nafion, there is phase segregation into hydrophilic and hydrophobic domains, ${ }^{7}$ provided by the absence of chemical cross-linking between polymeric chains, as described in the pioneering cluster-network model proposed by Gierke et al. ${ }^{8}$ Although this cluster-network model has been widely referenced, nowadays there is quite an agreement regarding its inaccuracy as it was based on the limited structure property information that was available at the time, ${ }^{9}$ and therefore, other more recent models are under debate. ${ }^{4,10-15}$

Up to now the unique nanostructure of Nafion has been used for producing polymer-metal hybrid nanomaterials by the use of the hydrated clusters of the polymer as reactive templates for the 
synthesis of metal nanoparticles (MNPs) such as Pt-NPs, Ag-NPs, Cd-NPs or PbS-NPs among others. ${ }^{16-20}$ It is noteworthy that most of the publications regarding this topic grounded the MNPs formation in the classic cluster-network model although many of these works reported NPs with a size larger than the typical cluster (about $4 \mathrm{~nm}$ ). Moreover, in many cases, NPs were mainly found on the surface or on the edges of the membrane instead of inside the matrix channels. ${ }^{19-20}$ In fact, the superficial distribution is usually observed for other ion-exchange matrices. ${ }^{21}$

This controversy can be explained taking into account that Nafion does not present a static morphology in aqueous environments as a consequence of the presence of extremely hydrophobic and extremely hydrophilic domains in the polymer backbone. In this sense, Gebel et al. proposed a water channel model as a conceptual description for the swelling and dissolution process. $^{22}$ In such qualitative model, the dry membrane is considered to contain isolated and spherical ionic clusters. With the absorption of water, the clusters swell to hold pools of water surrounded by ionic groups at the polymer-water interface in order to minimize the interfacial energy. As the water content increases, structural reorganization occurs to keep the specific surface area constant, and the onset of percolation is achieved by connecting cylinders of water between the swollen, spherical clusters. Finally, as the membrane "dissolves" into solution, either lamellar or film structures appear. Such structures are in agreement with Litt's and Kreuer's models describing Nafion as a multilayer structure where the ionic domains are defined as hydrophilic layers separated by thin hydrophobic PTFE-like domains. ${ }^{14-15}$ Thanks to these models, subsequent studies pointed to a nanoscale arrangement that could explain the excellent ion transport properties of the material. ${ }^{23-24}$

Recently, a short communication ${ }^{25}$ showed that the growth of Ag-NPs in Nafion-117 did not follow the usually found superficial distribution ${ }^{20}$ since in this case NPs were arranged inside the matrix in parallel stripes. This striped distribution is in agreement with the lamellar distribution of the hydrophilic and hydrophobic domains put forward by the last published morphological models of Nafion. ${ }^{4,15}$ Accordingly, Ag-NPs were revealing the hidden structured of the polymer in its hydrated form. Besides, it was also shown that after treating the nanocomposite samples with ultrasounds, the stripes were enlarged and more separated.

Yet, the aforesaid publication ${ }^{25}$ was focused on the description of the uncommon pattern and on the rationale behind the formation of stripes. Thus, a wide-ranging characterization aimed to determine the final properties of this particular nanocomposite was a pending issue. On the one hand, it was basic to improve the characterization of the nanoobjects and, on the other hand, it was crucial to investigate whether the stripes entail any substantial modification of the properties of the polymer or not.

Therefore, a complete chemical characterization of the immobilized Ag-NPs has been accomplished by the use of X-ray Absorption Near Edge Spectroscopy (XANES), X-Ray Powder Diffraction (XRD) and X-Ray Photoelectron Spectroscopy (XPS). Likewise, in order to assess how this unexpected patterning affects the electrical characteristics and the transport capabilities of the matrix (envisaging a possible use as PEMs), a thorough characterization of the Nafion-117/Ag-NPs nanocomposites has been performed by Impedance Spectroscopy (IS), membrane potential and mechanical stress analyses. 


\section{Experimental}

\section{Materials}

Nafion-117 membrane (DuPont, USA) was purchased from Sigma Aldrich. Chemical reagents such as $\mathrm{AgNO}_{3}$ and $\mathrm{NaBH}_{4}$ were all provided from Panreac except for $\mathrm{HNO}_{3}$ which was purchased from Fisher Chemical.

\section{Ag-NPs synthesis and post treatment}

Previous to the Ag-NPs synthesis, enough pieces of the commercial Nafion-117 membrane were pre-treated as described in $^{25}$ in order to eliminate any possible impurities and to enhance the ability of Nafion to absorb water (in comparison to the vacuum dried and as-received samples). ${ }^{26}$ Clean pieces were cut either in rectangular $(50 \mathrm{~mm} \times 20 \mathrm{~mm}$ ) or circular shape (diameter $6.2 \mathrm{~mm}$ ). Ag-NPs were synthesized inside the membranes by Intermatrix Synthesis (IMS), ${ }^{21,25,27-28}$ a two-step procedure consisting on i) loading the ionogenic groups with the metal precursor and ii) subsequent chemical reduction using $\mathrm{NaBH}_{4}$ solution $0.5 \mathrm{M}$ (Eq. 1 and Eq. 2):

$$
\begin{aligned}
& \left(\mathrm{R}^{-} \mathrm{SO}_{3}{ }^{-}\right) \mathrm{Na}^{+}+\mathrm{Ag}^{+} \rightleftharpoons\left(\mathrm{R}-\mathrm{SO}_{3}{ }^{-}\right) \mathrm{Ag}^{+}+\mathrm{Na}^{+} \\
& \left(\mathrm{R}-\mathrm{SO}_{3}{ }^{-}\right) \mathrm{Ag}^{+}+\mathrm{NaBH}_{4}+3 \mathrm{H}_{2} \mathrm{O} \rightarrow \rightarrow\left(\mathrm{R}-\mathrm{SO}_{3}{ }^{-}\right) \mathrm{Na}^{+}+(7 / 2) \mathrm{H}_{2}+\mathrm{B}(\mathrm{OH})_{3}+\mathrm{Ag}^{0}
\end{aligned}
$$

The $\mathrm{AgNO}_{3}$ concentration (either 2 or $15 \mathrm{mM}$ ) was chosen proportional to the wet weight of the membrane sample and to its ion-exchange capacity (namely $1.1 \mathrm{meq} / \mathrm{g}$ ) in order to assure an excess of $\mathrm{Ag}(\mathrm{I})$ in the loading solution. Some replicates (from 3 to 5 ) of each type were prepared.

As it was previously demonstrated, ${ }^{25,29}$ spherical Ag-NPs grow forming stripes which can be modulated by the use of ultrasounds. With the purpose of magnifying this effect, some of the soobtained nanocomposites were placed in a closed vessel with $10 \mathrm{~mL}$ bidistilled water and immersed for $1 \mathrm{~h}$ in an ultrasonic bath (Branson 1200, 20W).

The set of samples is summarized in Table 1. NO corresponds to the neat Nafion-117 membrane stored in a wet form and used as a comparative blank material. N1 correspond to the sonicated nanocomposite samples used for XRD, Inductively Coupled Plasma Mass Spectrometry (ICP-MS), XPS, Transmission Electron Microscopy (TEM) and electrochemical studies and mechanical behaviour studies. Finally, N2, N3, N4 and N5 correspond to groups of 5 circular samples posttreated and stored differently. Such samples were used for XANES experiments in order to evaluate any change in the speciation of $\mathrm{Ag}$ due to storage and/or aging. Note that the 5 samples labelled as N2 were just loaded with $\mathrm{Ag}(\mathrm{I})$ but not chemically reduced.

\section{Ag-NPs characterization}

The size, structure and distribution of Ag-NPs in the membranes were analysed by TEM with a JEM-1400 Jeol - TEM microscope. Before observation, samples were prepared to obtain transversal layers, which were afterwards sputtered with carbon. ${ }^{25}$

To determine the exact metal content in the as-prepared nanocomposites, they were analysed by ICP-MS (Agilent 7500). Samples of $1 \mathrm{~cm}^{2}$ were digested overnight with $1 \mathrm{~mL}$ of concentrated $\mathrm{HNO}_{3}$ $(65 \% \mathrm{w} / \mathrm{w})$ and then diluted 1:100 with bidistilled water. The resulting solutions were appropriately diluted for ICP-MS analyses (2 replicates). 
Nafion-117 surface modification due to the presence of the Ag-NPs was determined by XPS measurements, which were recorded with a Physical Electronic PHI 5700 spectrometer. Recorded spectra were fitted using Gauss-Lorentz curves following the methodology described elsewhere. ${ }^{30}$ Atomic concentration percentages of the elements in the membrane surfaces were determined considering the area sensitivity factor for the different measured spectral regions. ${ }^{31}$

Table 1. List of samples with details regarding synthesis, post-treatments and characterization techniques.

\begin{tabular}{|c|c|c|c|c|c|c|}
\hline Sample & Shape and Size & {$\left[\mathrm{AgNO}_{3}\right]^{\mathrm{a}}$} & {$\left[\mathrm{NaBH}_{4}\right]^{\mathrm{b}}$} & Sonication $^{c}$ & Storage & Characterized by \\
\hline N 0 & \multirow[b]{2}{*}{$\begin{array}{c}\text { Rectangular } \\
(50 \mathrm{~mm} \times 20 \mathrm{~mm})\end{array}$} & none & none & none & \multirow[b]{2}{*}{ in water } & $\begin{array}{c}\text { XRD } \\
\text { membrane potential } \\
\text { IS } \\
\text { stress-strain }^{d}\end{array}$ \\
\hline N1 & & $15 \mathrm{mM}$ & $0.5 \mathrm{M}$ & $1 \mathrm{~h}$ & & $\begin{array}{c}\text { XRD } \\
\text { membrane potential } \\
\text { IS } \\
\text { stress-strain }^{\text {d }} \\
\text { XPS }^{\text {e }} \\
\text { TEM } \\
\text { ICP-MS }\end{array}$ \\
\hline N2 & \multirow{4}{*}{$\begin{array}{c}\text { Circular } \\
\text { diameter }=6.2 \mathrm{~mm}\end{array}$} & \multirow{4}{*}{$2 \mathrm{mM}$} & none & none & $\begin{array}{c}\text { stored in } \\
\mathrm{AgNO}_{3} 2 \mathrm{mM}\end{array}$ & \multirow{4}{*}{ XANES } \\
\hline N3 & & & \multirow[t]{3}{*}{$0.5 \mathrm{M}$} & none & dried at air & \\
\hline N4 & & & & none & in water & \\
\hline N5 & & & & $1 \mathrm{~h}$ & in water & \\
\hline & $\begin{array}{l}\text { a: loading with } 25 \mathrm{~mL} A c \\
\text { : reduction with } 10 \mathrm{~mL} \\
\text { : samples were treated } \\
\text { : sample analysed in wale was analysed }\end{array}$ & $\begin{array}{l}{ }_{4} \mathrm{mM} \text { for } 24 \\
0.5 \mathrm{M} \text { ove } \\
\text { ultrasounds } \\
\text { as well as } \\
\text { and after }\end{array}$ & $\begin{array}{l}\text { ht. } \\
\text { ile submerg } \\
\text { ed at air. } \\
\text { tment with }\end{array}$ & $10 \mathrm{ml}$ of bidi & minutes. & \\
\hline
\end{tabular}

The surface of sample N1 was firstly analysed after drying the sample at vacuum conditions. Secondly, it was etched by Argon sputtering ( $4 \mathrm{kV}$ and $1.5 \mathrm{~mA}$ ) during 2 minutes in order to eliminate contributions associated to surface species. A more detailed explanation of the XPS protocol is available in ESI. (SI.1†).

XRD technique was used to obtain the crystalline structure of the obtained particles. XRD was performed using a PANanalytical X'Pert Pro automated diffractometer and identification of silver was done by comparing the characteristic peaks in the diffractograms and the databases. Details on the XRD protocol are available in ESI. (SI.2t)

In order to obtain a more detailed speciation of the Ag-NPs, XANES spectra were acquired at Ag K-edge (25514 eV) at CLAESS, the X-ray absorption spectroscopy beamline of ALBA synchrotron facility (Barcelona, Spain). Data normalization and linear combination was done using Athena package. ${ }^{32}$ Details on the XANES protocol are available in ESI. (SI.3T) 


\section{Membrane potential and transport number evaluation}

Membrane potential $\left(\Delta \Phi_{\mathrm{mem}}\right)$ is the stationary electrical potential difference at both sides of a membrane separating two solution of the same electrolyte but different concentration. $\Delta \Phi_{\text {mem }}$ was obtained by subtracting the electrode potential $\left(\Delta \Phi_{\text {elect }}\right)$ from the corresponding concentration potential values $(\Delta \mathrm{E})$ measured in a dead-end test-cell similar to that indicated elsewhere ${ }^{33}$ with $\mathrm{HCl}$ solutions and two $\mathrm{Ag} / \mathrm{AgCl}$ reversible electrodes (one at each membrane side) connected to a high impedance voltmeter (Yocogawa, $1 \mathrm{MW}$ internal impedance) (see Eq. 3):

$\Delta \Phi_{\text {mem }}=\Delta \mathrm{E}-\Delta \Phi_{\text {elect }}=\Delta \mathrm{E}-(\mathrm{RT} / \mathrm{F}) \ln \left(\mathrm{C}_{\mathrm{c}} / \mathrm{C}_{\mathrm{v}}\right)$

where $\mathrm{R}$ and $\mathrm{F}$ are the ideal gas and Faraday constants, $\mathrm{T}$ is the thermodynamic temperature of the system; concentrations, $C_{i}$, (instead of activities, $a_{i}$ ) can be used in the case of diluted solutions.

The measurements were performed keeping constant the concentration of the solution at one membrane side $\left(C_{c}=0.01 \mathrm{M}\right)$, and gradually changing the $\mathrm{HCl}$ concentration of the solution at the other membrane side $\left(C_{v}\right.$ from $10^{-3} \mathrm{M}$ to $\left.10^{-1} \mathrm{M}\right)$. To minimize the effect of concentration polarization next to the membrane, measurements were carried with solutions being stirred at a constant speed of $525 \mathrm{rpm} .^{34}$

Besides de membrane potential, another characteristic electrochemical parameters associated to the diffusive transport of ions through charged membranes is the ion transport number $\left(t_{i}\right)$ which represents the fraction of the total electric current transported by one ion $\left(t_{i}=I_{i} / I_{T}\right) \cdot{ }^{37}$ Taking into account the $t_{i}$ definition, the following relationship between the ions in the solutions should be fulfilled: $\Sigma_{i}\left(t_{i}\right)=1$. Thus, in the case of single salts, the following relation between cation $\left(t_{+}\right)$and anion ( $t_{-}$) transport numbers exists: $t_{+}+t_{-}=1$. For an ideal cation-exchange membrane, $t_{+}=1$, and the concentration potential measured between two solutions of concentrations $C_{1}$ and $C_{2}$, at both membrane sides, presents its maximum value at (Eq. 4): ${ }^{35}$

$\Delta \mathrm{E}_{\max }=-(2 \mathrm{RT} / \mathrm{F}) \ln \left(\mathrm{a}_{2} / \mathrm{a}_{1}\right) \approx-(2 \mathrm{RT} / \mathrm{F}) \ln \left(\mathrm{C}_{\mathrm{v}} / \mathrm{C}_{\mathrm{f}}\right)$

Cation transport number through a non-ideal membrane for a given pair of solution concentrations $\left(C_{v}, C_{f}\right)$ can be estimated by ${ }^{35}: t_{+}=\Delta E / \Delta E_{\max }$.

\section{Characterization by Impedance spectroscopy}

Impedance spectroscopy (IS) measurements for the system "Pt electrode // membrane // Pt electrode" were performed with a frequency response analyser (FRA, Solartron 1260, UK) controlled by a computer and connected to the respective Pt electrodes. 100 data points were collected for frequency ranging between $1 \mathrm{~Hz}$ and $10 \mathrm{MHz}$, with a maximum voltage of $10 \mathrm{mV} .^{36}$ Nanocomposite membranes kept in distilled water were softly dried with filter paper to avoid water dripping just before placing in the measuring cell.

\section{Mechanical properties analysis}

The mechanical behavior of the membranes and of the nanocomposites (in wet form and after drying at air) was determined from the stress-strain curves using a force meter Mark-T ES20 connected to a computer, with a maximum tension of $100 \mathrm{~N}$, length accurate of $\pm 0.01 \mathrm{~mm}$ and a strength rate of $10 \mathrm{~mm} / \mathrm{s}$. 


\section{Results and discussion}

\section{Ag-NPs characterization}

As shown in Fig. 1a, Ag-NPs obtained by IMS in cleaned and hydrated Nafion-117 membranes were found all over the polymeric matrix following an aligned pattern in the form of stripes that has been discussed earlier ${ }^{25}$ and which is in agreement with the lamellar distribution of the hydrophilic and hydrophobic domains of the matrix. However, this situation is very different to that of NPs made of other metals obtained in an almost identical manner in the same polymeric matrix. ${ }^{28}$ For instance, Pd-NPs were mainly found on the edge of samples. ${ }^{21}$ The conventional explanation for such situation is the hindrance of the negatively charged reducing agent $\left(\mathrm{BH}_{4}{ }^{-}\right)$to penetrate the polymeric matrix bearing negatively charged groups $\left(-\mathrm{SO}_{3}{ }^{-}\right)$, thus concentrating the amount of NPs on the sample surface. Such explanation could be valid at limited extent for Nafion117/Ag-NPs since in Fig. 1b it is perceived that inner Ag-NPs (Fig. 1b) were found to be smaller $(10.1 \pm 0.2 \mathrm{~nm})$ than those on the surface (Fig. 1b). As a matter of fact, this was not a completely unexpected result if we consider that, during synthesis, a larger amount of reactants can easily and fast react in the superficial part of the membrane producing, therefore, larger Ag-NPs.

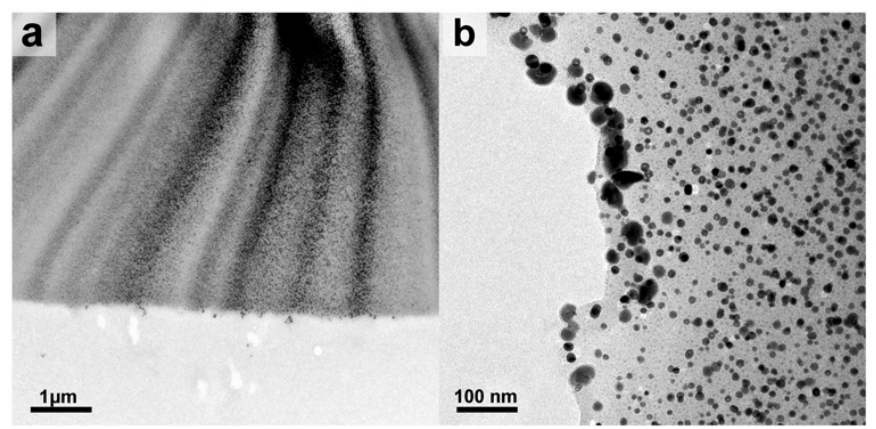

Figure 1. TEM images of ultra-thin slices of Nafion-117/Ag-NPs: (a) general view of stripes, (b) surface edge. Figure 1a is reproduced by permission of The Royal Society of Chemistry ${ }^{25}$. (http://pubs.rsc.org/en/content/articlelanding/2014/cc/c4cc01285b\#!divAbstract).

\section{Chemical composition characterization}

The average silver content in the nanocomposite sample N1 was found to be $51.8 \pm 1.5 \mathrm{mg} \mathrm{Ag} / \mathrm{g}$ dry membrane (ca. $5 \mathrm{wt} \%$ ) as determined by ICP-MS. Note that since samples were digested in acid media prior to the analysis, this amount takes account for the total silver found through the membrane, disregarding its oxidation state or its location in the sample (inner part or surface).

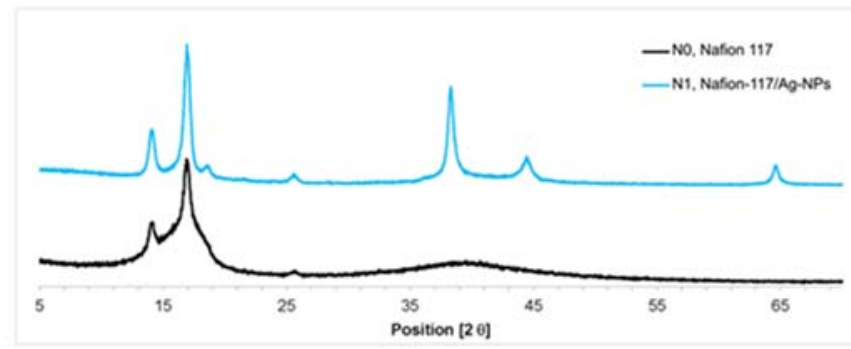

Figure 2. X-Ray diffractograms of neat Nafion-117 (NO) and Nafion-117/Ag-NPs sample (N1). 
The X-Ray diffractograms of NO and N1 samples are presented in Fig. 2. The broad signals or haloes located between $2 \theta=12$ and $2 \theta=18$ are typical of the neat Nafion- 117 corresponding to the crystalline and amorphous part of the polymer and are in agreement with those found in the literature. ${ }^{37-39}$ The broad signal of the matrix made it difficult to clearly identify any peak corresponding to minor components but it can be clearly observed that sample N1 contained metallic silver as it can be deduced from the peaks found at the following values of $2 \theta: 36.5,44.5$ and 65 (approximately) which correspond to reflections of the face-cantered cubic Ag crystalline lattices (111), (200) and (220). Since Ag peaks are broadened, it is possible to affirm that $\mathrm{Ag}(\mathrm{I})$ ions were successfully reduced to Ag-NPs at high extent. ${ }^{40-43}$

Besides, the analysis by XPS provided more detailed information on silver speciation and location, since this technique is surface sensitive and gives qualitative and quantitative information of the elements present in a thickness less than $10 \mathrm{~nm}$ through the study of the binding energy. ${ }^{44}$

As it can be observed in Fig. 3a, the solid line (sample N1) shows two symmetrical peaks in the binding energy of $\mathrm{Ag}^{0} 3 d_{5 / 2}$ (ca. $368 \mathrm{eV}$ ) and of $\mathrm{Ag}^{0} 3 d_{3 / 2}$ (ca. $375 \mathrm{eV}$ ). Both peaks are usually attributed to $\mathrm{Ag}$ what would be consistent with the conclusion provided by the analysis of the XRD diffractograms. The silver content on the surface was $13 \%$ (in atomic concentrations).
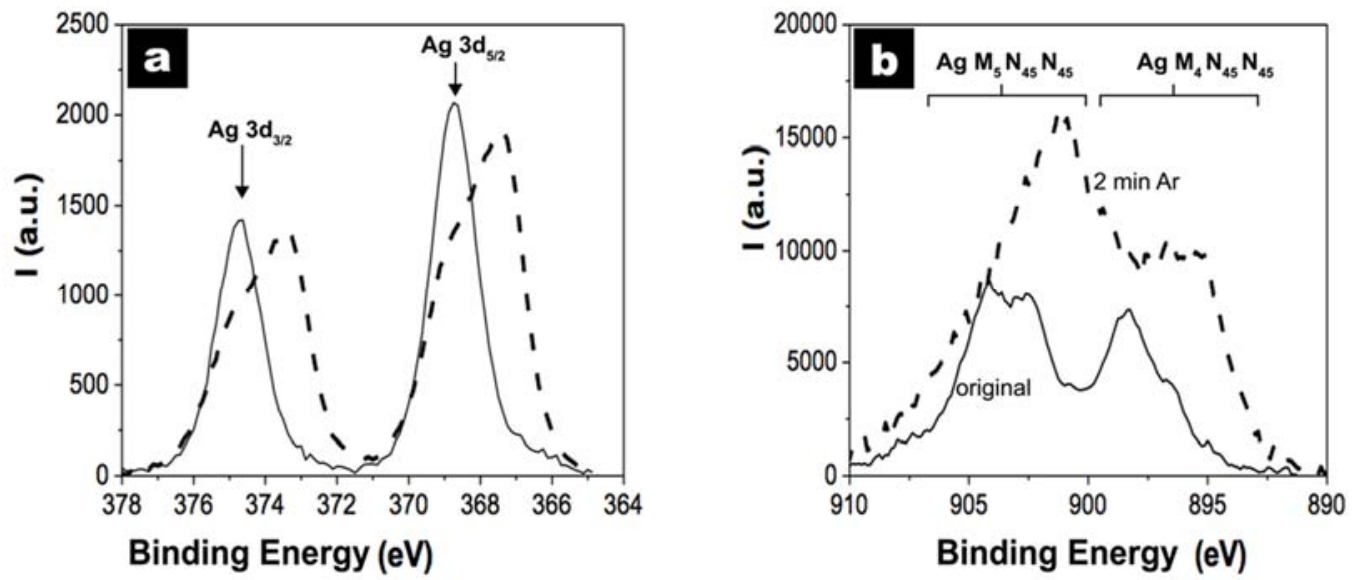

Figure 3. (a) XPS spectra for Ag 3d core level and (b) Ag MNN Auger spectra. Solid line corresponds to the original Nafion-117/Ag-NPs (N1) membrane while the dashed line corresponds to the same sample upon surface erosion by 2 minutes Ar sputtering.

XPS also allows depicting the oxidation state although with some limitations. In this particular case, the binding energies for oxidized spices of $\mathrm{Ag}$ are reported as almost coincident with the one for the reduced form and there is some dispersion of binding energy values and even superpositions. ${ }^{44}$ In order to evaluate the Ag speciation, a piece of sample N1 was sputtered by a Ar plasma during 2 minutes before measuring. This etching procedure is used in subsurface analysis and allows removing the most superficial part of the sample, and therefore the contribution of the outer species to the analysis. ${ }^{45}$ Some differences were observed when the spectra of the original sample (solid line) and the etched sample (dashed line) were compared: for the original sample peaks are situated at slightly higher binding energies. In other words, upon etching, both $\mathrm{Ag}^{0} 3 d_{3 / 2}$ and $\mathrm{Ag} 3 d_{5 / 2}$ peaks were evidently shifted to lower binding energies (373 and $367 \mathrm{eV}$, respectively) what is compatible with the removal of a surface layer rich in oxidized $\mathrm{Ag}(\mathrm{I})$ species and the revealing of reduced $\mathrm{Ag}^{0}$ on the subsurface of the sample. Similar oxide removal via Ar sputtering has been reported earlier for silver containing cermets. ${ }^{45}$ 
The two Ag MNN Auger spectra plotted in Fig. 3b indicate the same effect even in a clearer way since energy differences are higher as it usually happens. ${ }^{46}$

Thus, from XPS results it came that N1 sample contained zero-valent silver as well as some oxidized species which could be located on the surface of the membrane. The coexistence of $\mathrm{Ag}^{0}$ and $\mathrm{Ag}(\mathrm{I})$ species was quite surprising taking into account that the reducing step was performed with a strong reducing agent at high concentration $\left(0.5 \mathrm{M} \mathrm{NaBH}_{4}\right)$. One can think that $\mathrm{Ag}(\mathrm{I})$ species could appear either due to sample incomplete reduction or because of post-oxidation.

In order to obtain a more specific analysis and ensure the chemical composition of silver Nafion117 , synchrotron experiments were carried out comparing the spectra of samples with the spectra of pure standards, concretely, metallic silver foil $\left(\mathrm{Ag}^{\circ}\right)$ and solid inorganic salts such as $\mathrm{Ag}_{2} \mathrm{O}$ and $\mathrm{AgNO}_{3}$. Note that the election of these two salts is justified since $\mathrm{AgNO}_{3}$ is the precursor used to carry out the Ag-NPs synthesis and $\mathrm{Ag}_{2} \mathrm{O}$ is the most-probable composition derived of the interaction of ionic silver with air.

Regarding the synchrotron samples, the selection was done following these premises:

i. Sample N2: unreduced Nafion-117/ $\mathrm{Ag}(\mathrm{I})$ stored in $\mathrm{AgNO}_{3}$ solution instead of water to provide information about the $\mathrm{Ag}(\mathrm{I})$ species immobilised in the polymer.

ii. Sample N3: Nafion-117/Ag-NPs without sonication and dried at air just after synthesis to confirm the possible oxidation of $\mathrm{Ag}$ due to air when in the nanocomposite.

iii. Sample N4: Nafion117/Ag-NPs without sonication and stored in water to determine the possible oxidation of $\mathrm{Ag}$ when in water.

iv. Sample N5: the analogous to N1.

After carrying out the XANES analyses, data treatment and curve deconvolution (see Fig. 4), the obtained results together with the R-factor values (lower than 0.003 which indicate good fits for all the samples) are reported in Table 2.

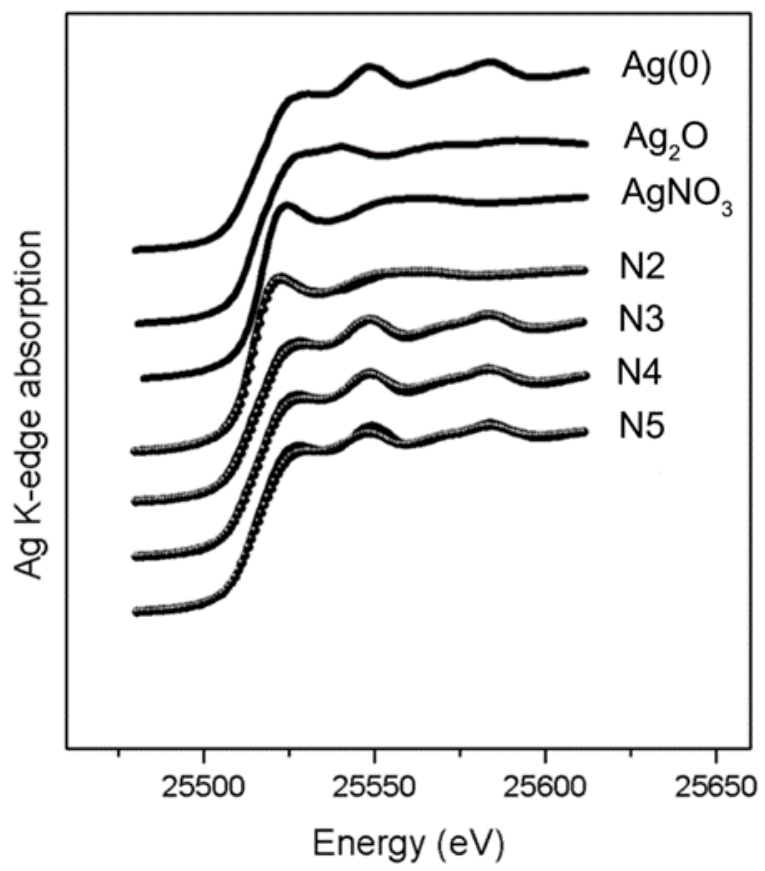

Figure 4. Normalized Ag K-edge XANES spectra for samples N2-N5 (straight line) and the corresponding fit (spheres). References used for the linear combination ( $\mathrm{Ag}$ foil, $\mathrm{Ag}_{2} \mathrm{O}$ and $\mathrm{AgNO}_{3}$ ) are shown above. 
Table 2. Relative amount of $\mathrm{Ag}^{0}, \mathrm{Ag}_{2} \mathrm{O}$ and $\mathrm{AgNO}_{3}$ in each sample from $\mathrm{XANES}$ data treatment.

\begin{tabular}{|c|c|c|c|c|}
\hline Sample & $\mathbf{A g}^{\mathbf{0}}(\%)$ & $\mathbf{A g}_{\mathbf{2}} \mathbf{O}(\%)$ & $\mathrm{AgNO}_{3}(\%)$ & $\mathrm{R}$-factor \\
\hline $\mathrm{N} 2$ & $0 \pm 2$ & $6 \pm 2$ & $94 \pm 2$ & 0.0010 \\
\hline $\mathrm{N} 3$ & $87 \pm 2$ & $6 \pm 2$ & $7 \pm 2$ & 0.0007 \\
\hline $\mathrm{N} 4$ & $84 \pm 3$ & $7 \pm 3$ & $9 \pm 2$ & 0.0011 \\
\hline $\mathrm{N} 5$ & $68 \pm 5$ & $32 \pm 4$ & $0 \pm 2$ & 0.0026 \\
\hline
\end{tabular}

For all the cases, excepting N2, the most of the silver in the samples was found in the form of metallic zero-valent silver $\left(\mathrm{Ag}^{0}\right)$ although different proportions of oxidized silver were also detected. By direct observation of Fig. 4 it is clearly seen that curve for sample N2 highly coincided with that of $\mathrm{AgNO}_{3}$, what is perfectly coherent since for this sample $\mathrm{Ag}(\mathrm{I})$ ions did not undergo any reduction process. However, regarding the related values in Table 2 some $\mathrm{Ag}_{2} \mathrm{O}$ was also unexpectedly detected (4-8\%) testifying for a possible ambient oxidation.

Otherwise, the curves of samples N3, N4 and N5 were closer to the curve of $\mathrm{Ag}^{0}$ foil although not exactly. Actually, the lowest percentage of metallic silver $(68 \pm 5 \%)$ matched with sample N5 that underwent sonication (being the most similar sample in relation to the rest of chemical characterizations). In this case, the amount of $\mathrm{Ag}_{2} \mathrm{O}$ reached the $32 \%$, so the ratio $\mathrm{Ag}: \mathrm{Ag}_{2} \mathrm{O}$ was about 2:1. These results accounted for the higher strength of the ultrasound aging treatment compared with the air-drying treatment (N3) and the storage in water (N4). For the last two samples (N3 and N4) the amount of reduced silver was in the range $84-87 \%$.

Note that these results are in agreement the previous characterisations: i) XRD values of $2 \theta$ for the sonicated sample N1 mainly corresponded to metallic silver which is, in fact, the main component of every sample except N2; ii) XPS demonstrated that oxidized silver could be found in the samples and, specifically, $\mathrm{Ag}_{2} \mathrm{O}$ species were more likely to be found on the surface due to the reaction with atmospheric oxygen. From the results it also comes that the oxidation process seemed to have been enhanced by the use of sonication as aging treatment.

The use of XANES allowed a better understanding of the sample aging, providing more detailed information. For the other nanocomposites (N2, N3, N4), the percentage of $\mathrm{AgNO}_{3}$ was marginal compared to metallic silver, so it would not have been detected by XRD either because the amount of this compound were too low to show peaks or it were not crystalline. ${ }^{40}$

\section{Electrochemical characterization}

The cation transport number through a membrane is an electrochemical parameter of crucial interest for electrochemical systems. ${ }^{47,48}$ In this case, the cation transport number corresponded to the proton transport $\left(t_{H+}\right)$, since it was determined from the membrane potential $\left(\Delta \Phi_{\text {mem }}\right)$ measurements performed with $\mathrm{HCl}$ solutions. Fig. 5 shows the variation of the membrane potential with the ratio of the $\mathrm{HCl}$ at both sides of the Nafion-117 (NO) and Nafion-117/Ag-NPs (N1) membranes, together with the theoretical value of an ideal negatively charged (dashed line).

The first thing to notice is that there were no differences between the behaviour of a negatively charged ideal cation exchanger and any of the samples. The fitting of the experimental data identified the following values for the transport number: $\mathrm{t}_{\mathrm{H}+(\mathrm{N})}=0.98 \pm 0.02$ and $\mathrm{t}_{\mathrm{H}+(\mathrm{N} 1)}=0.96 \pm 0.03$. 


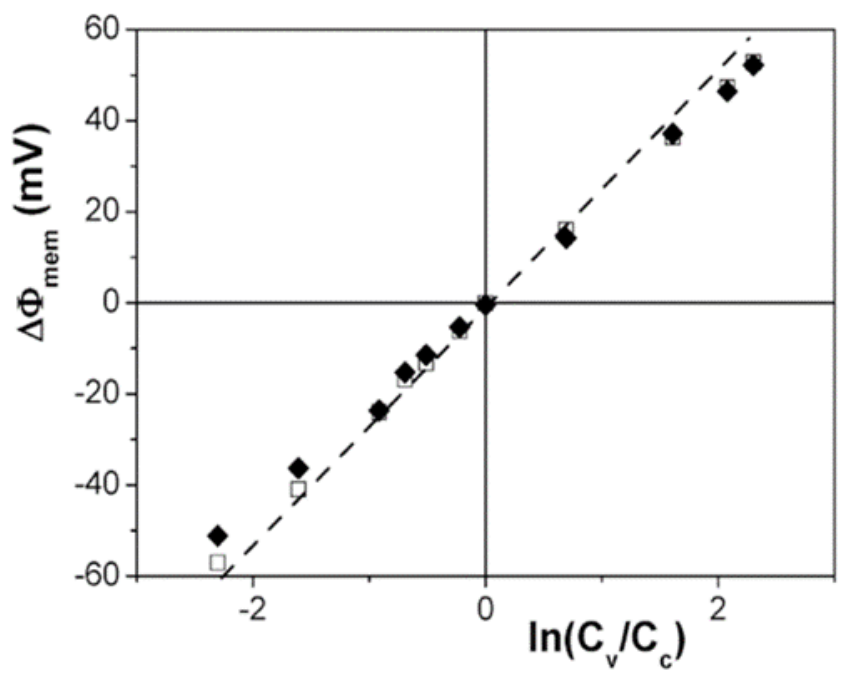

Figure 5. Membrane potential as a function of $\mathrm{C}_{\mathrm{HCl}}$ ratio for samples NO ( $\square$ ) and N1 (

Note that the value of $t_{H_{+}}$obtained for the Nafion-117 membrane was similar to that reported in the literature for this membrane and for Nafion-112, ${ }^{48,49}$ which supports the adequacy of the electrochemical results obtained in this work with both samples.

Accordingly, the presence of Ag-NPs in the nanocomposite structure hardly affected the mechanism of proton transport across Nafion membranes, supporting the suitability of these nanocomposites for their use in electrochemical-based devices.

Membrane electrical conductivity is another key-parameter commonly used for the characterization of PEMs, and it is determined from IS measurements. Fig. 6 displays the typical Bode plot ( $Z_{\text {real }} v s$ frequency) for the unmodified Nafion-117 (sample NO) and for the nanocomposite (sample N1), both in the hydrated state.

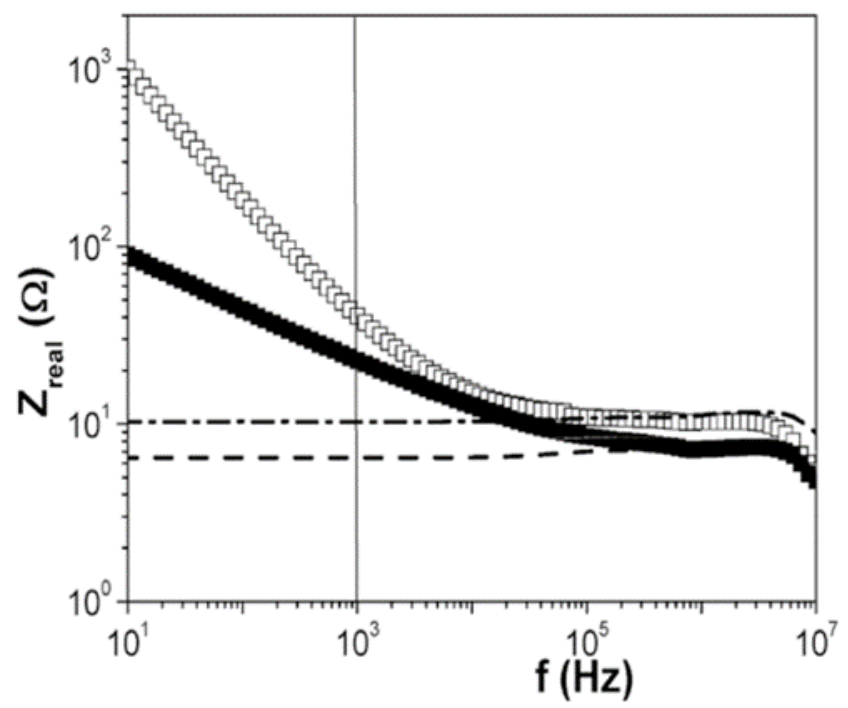

Figure 6. Variation of the real part of the impedance $\left(\mathrm{Z}_{\text {real }}\right)$ with frequency for NO $(\square)$ and $N 1(\diamond)$, and the values for two resistors of $10 \Omega(\cdot-$ --) and $5 \Omega(---)$. 
As it can be observed in Fig. 6, curves are not dramatically different and there is a non-negligible resistance for the nanocomposite sample testifying that the stripes Ag-NPs are not connected forming an electron-conduction path what would prevent its use as PEM. ${ }^{50}$

Regarding the plot, at frequencies above $1 \mathrm{kHz}$ (the part of the plot which is usually attributed to the impedance associated with the membrane), there are no significant differences between the two membranes even if the resistance of the nanocomposite is slightly lower above $100 \mathrm{kHz}$. For the sake of comparison, in Fig. 6 there are also shown, by dashed and dot-dashed lines, the experimental values obtained for two pure resistances with nominal values of $5 \Omega$ and $10 \Omega$, respectively. As it can be observed, there is a good correlation between these values and those obtained for the membranes in the frequency range corresponding to the material itself. NO sample (without Ag-NPs) highly coincides with the $10 \Omega$ resistance, whereas N1 sample (with Ag-NPs) to the $5 \Omega$ resistance, indicating a reduction in the electrical resistance of the Ag-NPs modified sample and, consequently, an increase in its electrical conductivity.

By contrast, values plots clearly differ in the low frequency range, between $1 \mathrm{~Hz}$ and $1 \mathrm{kHz}$, being this region commonly associated with the electrode-membrane interface. As it is shown, in the low frequency range, impedance of the Ag-NPs modified membrane (N1) decreases compared to the non-modified sample (NO) so the surface of both samples should be different, what is in clear agreement with the TEM observations as Ag-NPs decorate the surface of the nanocomposite. Thus, Ag-NPs improve the electrical contact between the membrane and the electrode.

\section{Mechanical properties}

In order to evaluate if the presence of such Ag-NPs patterns could affect other properties of the membrane (i.e. its mechanical behaviour), the mechanical characterization of different samples was evaluated through stress-strain curves and results are shown in Fig. 7. Samples NO and N1 were tested in both hydrated $(h)$ and dry $(d)$ states and clear differences between the four specimens were detected.
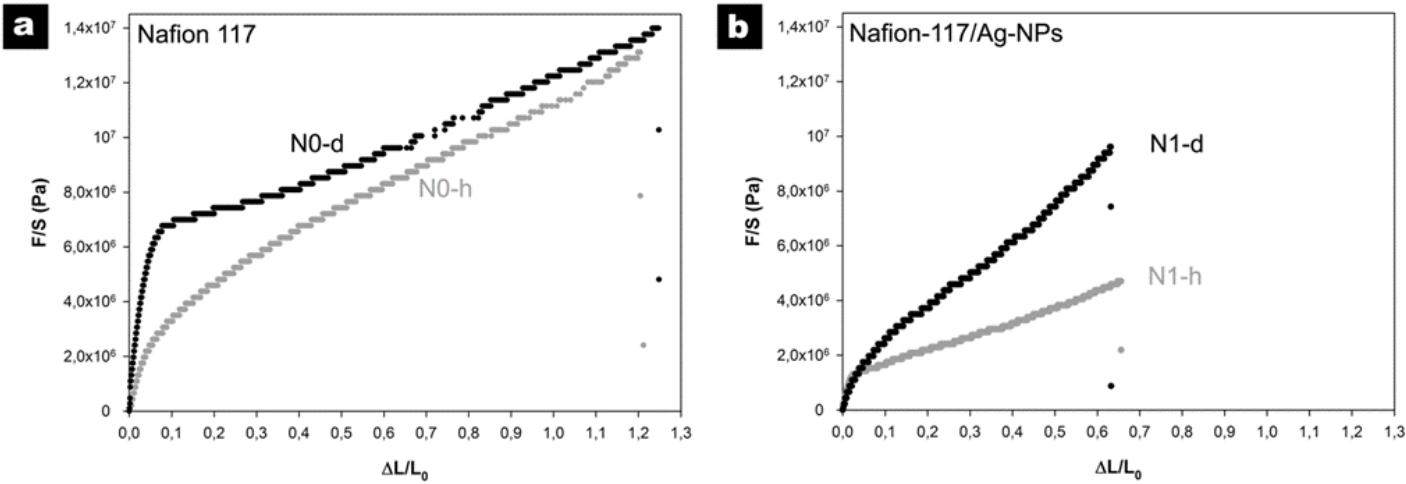

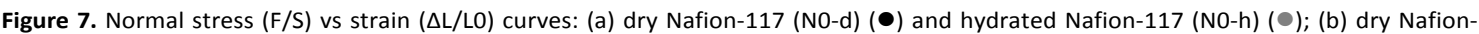
$117 / \mathrm{Ag}(\mathrm{N} 1-\mathrm{d})(\bullet)$ and hydrated Nafion-117/Ag (N1-h) (๑).

First, it is clearly seen that the inclusion of Ag-NPs in the membranes undoubtedly lowered the mechanical resistance of the material since the elongation at break was almost halved for the samples containing Ag-NPs compared with the unmodified Nafion-117 samples. That happened disregarding the level of hydration because the values were very similar for each couple of samples $(\mathrm{NO}-\mathrm{h} \approx \mathrm{NO}-\mathrm{d} \approx 1.2$ and $\mathrm{N} 1-\mathrm{h} \approx \mathrm{N} 1-\mathrm{d} \approx 0.6)$. Such dramatic reduction of mechanical 
properties implies the existence of structural irregularities which promote the separation of the polymer chains when a distance threshold is reached.

Second, when comparing the profiles of the stress-strain curves it is possible to see that the trends of the samples were completely opposite: NO-d and NO-h converged before breaking whereas N1$\mathrm{d}$ and N1-h diverged.

At low deformation values, NO-d and NO-h were dissimilar, NO-d being stiffer. The Young's modulus of NO-d was $(1.08 \pm 0.02) \cdot 10^{8} \mathrm{~Pa}$ compared with $(6.33 \pm 0.12) \cdot 10^{7} \mathrm{~Pa}$ of NO-h and close to the values reported previously. ${ }^{39}$ The yield point of NO-d was much higher in the domain of stress with an abrupt transition between the elastic and the plastic regions. Such differences are attributable to the plasticizing effect of the water embedded in the membrane structure. However, the higher the deformation, the higher the coincidence of the samples what is reasonable: as molecular chains are progressively separated due to extension, water molecules are no longer able to bind the polymer, cohesion is reduced and stress values are more similar, just to the point that breaking happens almost at the same elongation and the same stress. Conversely, for nanocomposite samples the behaviour is totally different: at low elongations both N1 samples were very similar showing a similar Young's modulus which was lower than that of NO-h but they became different progressively up to the breaking. In this case, Ag-NPs generate a plastic deformation effect in the whole interval although it is more important at low deformations $(<0.3)$ and for the dried sample (N1-d). Such effect could be explained taking into account that the polymeric chains lose the ability to move freely due to a strong interaction with the embedded nanoparticles. Similar effects have been reported previously for other systems (i.e. SPEEK-Cu, Nafion- silicates or Nafion-TiO2) pointing to a sort of cross-linking effect between polymer chains and NPs. ${ }^{27,39,51}$

Accordingly, it is possible to state that the incorporation of Ag-NPs weakens the material, however, since membranes in electrochemical devices are not usually subjected to high mechanical stress (except electrode compression in PEMFCs application), the applicability of the developed materials for such electrochemical applications may not be strongly hindered by the lower mechanical performance.

\section{Conclusions}

The use of the IMS technique in Nafion-117 membranes has led to the formation of Ag-NPs (with diameters around $10 \mathrm{~nm}$ ), which are organized as uncommon stripes through the polymeric matrix. The characterization of this new material in regards to its possible application has been assessed and, under such view, morphological, physicochemical and electrochemical characterizations of Nafion-117 and Nafion-117/Ag-NP samples have been evaluated.

The presence of silver was confirmed by the analysis of a digested sample by ICP-MS that determined that the content of silver was ca. $5 \%$ weight of dry sample. Regarding the oxidation state of such metal, XRD was only able to ascertain the existence of $\mathrm{Ag}^{0}$ nanocrystals whereas the combination of XPS technique and XANES identified both $\mathrm{Ag}^{\circ}$ and $\mathrm{Ag}(\mathrm{I})$ species, being the last species located on the very surface and easily removed by Ar etching. Synchrotron experiments proved the existence of different types of oxidized silver, being the sample aged by sonication the one with a lower amount of zero-valent silver $\left(\mathrm{Ag}^{0} \mathrm{ca} .68 \%\right)$, but also revealing that some $\mathrm{Ag}_{2} \mathrm{O}(4-$ $8 \%)$ is present even in samples only loaded with $\mathrm{AgNO}_{3}$ and not chemically reduced. Taking into 
account both characterizations, it is expected that oxidized Ag-NPs were located only on the very surface due to the effect of atmospheric oxygen.

From the electrochemical studies, it was proved that the presence of Ag-NPs do not hinder the ion transport properties of the matrix as there were no differences between the transport number of the Nafion-117 membrane and the Ag-NPs loaded sample, being both very similar to an ideal negative ion-exchanger. Furthermore, from the results obtained by IS measurements, it is also possible to state that electrical conductivity (a desirable property in most of the electrochemical devices) has been slightly enhanced in the sample modified with Ag-NPs.

From elongation tests, it has been found that the inclusion of Ag-NPs clearly affects the mechanical properties of Nafion in two ways: Ag-NPs provoke a plasticization additional to that of water and, more important, they reduce the mechanical resistance of the membrane (ca. 50\%), probably due to a loss of the free-movement of the polymeric chains, derived from a strong interaction with the embedded nanoparticles. This result prevents the use of such membranes in those applications of high mechanical stress.

Overall, the results indicate that the developed material may be as suitable as Nafion to be used in electrochemical devices such PEMs although the existence of Ag-NPs could be advantageous for other devices such as sensors, either electrochemical or based in surface enhanced Raman scattering (SERS), where the properties of Ag-NPs might increase the response signal or reduce the response time..$^{40,52,53}$

\section{Acknowledgements}

We thank to the CICYT (MINECO, Spain, project CTQ/2011-27770 FEDER funds) for financial support and Ing. M.V. Martinez de Yuso, Servicios Generales de Investigación de la Universidad de Málaga (XPS Laboratory). Many thanks are given to Servei de Microscòpia from Universitat Autònoma de Barcelona. Wojciech Olszewski and Carlo Marini are acknowledged for their help during the data acquisition at CLAESS beamline from ALBA synchrotron light source and in the XANES data treatment.

\section{References}

1 K.A. Mauritz and R.B. Moore. Chem. Rev., 2004, 104, 4535-85.

2 C. Heitner-Wirguin. J. Membrane Sci., 1996, 120, 1-33.

3 H-C. Chien, L-D. Tsai, C-M. Lai, J-N. Lin, C-Y. Zhu and F-C. Chang. J. Power Sources, 2013, 226, 87-93.

4 P.V. Komarov, I.N. Veselov, P.P. Chud and P.G. Khalatur. Soft Matter, 2010, 6, 3939-3956.

5 K. Tyszczuk-Rotko, I. Bęczkowska, M. Wójciak-Kosior and I. Sowa. Talanta, 2014, 129, 384-391.

6 H. Huang, Y. Yu and K.H. Chung. Int. J. Hydrogen Energy, 2014, 39, 13832-13837.

7 S.C. DeCaluwe, P.A. Kienzle, P. Bhargava, A.M. Baker and J.A. Dura. Soft Matter, 2014, 10, 5763-5776.

8 T.D. Gierke, G.E. Munn and F.C. Wilson. Morphology of Perfluorosulfonated Membrane Products. In Perfluorinated Ionomer Membranes, American Chemical Society: 1982; Vol. 180, 195-216.

9 J.A. Elliott, D. Wu, S.J. Paddison and R.B. Moore. Soft Matter, 2011, 7, 6820-6827

10 M. Fujimura, T. Hashimoto and H. Kawai. Macromolecules, 1982, 15, 136-144.

11 G. Gebel and J. Lambard. Macromolecules, 1997, 30, 7914-7920.

12 H.G. Haubold, T. Vad, H. Jungbluth and P. Hiller. Electrochim. Acta, 2001, 46, 1559-1563.

13 L. Rubatat, A.L. Rollet, G. Gebel and O. Diat. Macromolecules, 2002, 35, 4050-4055.

14 M.H. Litt. A Reevaluation of Nation morphology. American Chemical Society Polymer Preprints 1997, 38.

15 K-D Kreuer and G. Portale. Adv. Funct. Mater., 2013, 23, 5390-5397. 
16 S. Wang, P. Liu, X. Wang and X. Fu. Langmuir, 2005, 21, 11969-11973.

17 P. Bertoncello, M. Peruffo and P.R. Unwin. Chem. Commun., 2007, 16, 1597-1599.

18 H.W. Rollins, T. Whiteside, G.J. Shafer, J-J. Ma, M-H. Tu, J-T. Liu, D.D. DesMarteau and Y-P. Sun. J. Mater. Chem., 2000, 10, 2081-2084.

19 H.W. Rollins, F. Lin, J. Johnson, J-J. Ma, J.T. Liu, M.H. Tu, D.D. DesMarteau and Y-P. Sun. Langmuir. 2000, 16, 8031-8036.

20 A. Sachdeva, S. Sodaye, A.K. Pandey and A. Goswami. Anal. Chem. 2006, 78, 7169-7174.

21 P. Ruiz, M. Muñoz, J. Macanás, C. Turta, D. Prodius and D.N. Muraviev. Dalton T., 2010, 39, 1751-1757.

22 G. Gebel. Polymer, 2000, 41, 5829-5838.

23 K. Schmidt-Rohr and Q.Chen. Nat. Mater., 2008, 7, 75-83.

24 O. Diat and G. Gebel. Nat. Mater., 2008, 7, 13-14.

25 B. Domènech, M. Muñoz, D.N. Muraviev and J. Macanás. Chem. Commun., 2014, 50, 4693-4695.

26 R. B. Moore and C. R. Martin. Macromolecules, 1988, 21, 1334-1339.

27 D.N. Muraviev, J. Macanás, M. Farre, M. Muñoz and S. Alegret. Sensor. Actuat B-Chem., 2006, 118, 408417.

28 B. Domènech, M. Muñoz, D.N. Muraviev and J. Macanás. Catal. Today, 2012, 193, 158-164.

29 R. Boistelle and J. P. Astier. J. Cryst. Growth, 1988, 90, 14-30.

30 M. J. Ariza, J. Benavente and E. Rodríguez-Castellón, The Capability of X-ray Photoelectron Spectroscopy in the Characterization of Membranes: Correlation between Surface Chemical and Transport Properties in Polymeric Membranes, in Handbook of Membranes: Properties, Performance and Applications, pp. 257290. Nova Science Publishers, Inc., New York, 2009.

31 J. Lukas and B. Jezek. Collection Czech. Chem. Commun., 1983, 48, 2909-2913.

32 B. Ravel and M. Newville. J. Synchrotron Radiat., 2005, 12, 537-541.

33 V. Romero, V. Vega, J. García, V.M. Prida, B. Hernando and J. Benavente. J. Colloid Interf. Sci., 2012, 376, 40-46.

34 L. Peláez, M.I. Vázquez and J. Benavente. Ceram. Int., 2010, 36, 797-801.

35 N. Lakshminarayanaiah. Transport Phenomena in Membranes, Academic Press, New York, 1969.

36 E. Maya, J. Benavente and J. de Abajo. Mater. Chem. Phys., 2012, 131, 581-588.

37 J. Jalili, S. Borsacchi and V. Tricol. J. Membrane Sci., 2014, 469, 162-173.

38 B.R. Matos, R.A. Isidoro, E.I. Santiago, M. Linardi, A.S. Ferlauto, A.C. Tavares and F.C. Fonseca. J. Phys. Chem. C, 2013, 117, 16863-16870.

39 J-W. Lee, Y-T. Yoo and J.Y. Lee. Macromol. Res., 2015, 23, 167-176.

40 W. Wang, M. Yang, Z. Wang, J. Yan and C. Liu. RSC Adv., 2014, 4, 63079-63084.

41 S. Agnihotri, S. Mukherji and S. Mukherji. RSC Adv., 2014, 4, 3974-3983.

42 N. Atar, T. Eren, B. Demirdögen, M.H. Yola and M.O. Çaglayan. Ionics, 2015, 21, 2285-2293.

43 S. Wang, P. Liu, X. Wang and X. Fu. Langmuir, 2005, 21, 11969-11973.

44 A.M. Ferraria, A.P. Carapeto and A.M. Botelho do Rego. Vacuum, 2012, 86, 1988-1991.

45 R. Romero, F. Martin, J.R. Ramos-Barrado and D. Leinen. Surf. Interface Anal., 2010, 42, 1172-1175.

46 D. Briggs and J.T. Grant (editors). Surface Analysis by Auger and X-Ray Photoelectron Spectroscopy. Chichester: IM Publications: 2003.

47 Choi, N.H. Jalani, and R. Datta. J. Electrochem. Soc., 2005, 152, 123-130.

48 V. Romero, M.V. Martínez de Yuso, A. Arango, E. Rodríguez-Castellón and J. Benavente. Int. J. Electrochem., 2012, 2012, Article ID 349435, 1-10.

49 I.A. Stenina, Ph. Sistat, A.I. Rebrov, G. Pourcelly and A.B. Yaroslavtsev. Desalination, 2004, 170, 49-57.

50 Y. Liu, T. Nguyen, N. Kristian, Y. Yu and X. Wang. J. Membr. Sci., 2009, 330, 357-362.

51 K. Ketpang, S. Shanmugam, C. Suwanboon, N. Chanunpanich and D. Lee. J. Membr. Sci., 2015, 493, 285-298.

52 V. Halouzka, P. Jakubec, C. Gregor, D. Jancik, K. Papadopoulos, T. Triantis and J. Hrbac. Chem. Eng. J., 2010, 165, 813-8118.

53 I. Fratoddi, A. Bearzotti, I. Venditti, C. Cametti and M.V. Russo. Sensor Actuat. B-Chem., 2016, 225, 96-108. 


\section{SUPLEMENTARY INFORMATION}

\section{Chemical and electrochemical characterization of Nafion containing silver nanoparticles in stripe-like distribution}

B. Domènech, V. Romero, M.I. Vázquez, M. Ávila, J. Benavente, M. Muñoz and J. Macanás

\section{SI.1. XPS measurements detailed protocol.}

Nafion-117 surface modification due to the presence of the Ag-NPs was determined by XPS measurements, which were recorded with a Physical Electronic PHI 5700 spectrometer with X-ray $\mathrm{MgK}_{\mathrm{a}}$ radiation (300W, $15 \mathrm{kV}, 1253.6 \mathrm{eV}$ ) as the excitation source. High-resolution spectra were recorded at a take-off angle of 450 by a concentric hemispherical analyser operating in the constant pass energy mode at $29.35 \mathrm{eV}$, using a $720 \mathrm{~mm}$ diameter analysis area; the $\mathrm{Au} 4 \mathrm{f}_{7 / 2}$ line was recorded with $1.16 \mathrm{eV}$ FWHM at a binding energy of $84.0 \mathrm{eV}$. The spectrometer energy scale was calibrated using $\mathrm{Cu} 2 \mathrm{p}_{3 / 2}, \mathrm{Ag} 3 \mathrm{~d}_{5 / 2}$, and $\mathrm{Au} 4 \mathrm{f}_{7 / 2}$ photoelectron lines at $932.7,368.3$, and 84.0 $\mathrm{eV}$, respectively. Charge referencing was done against $-\mathrm{CF}_{2^{-}}$carbon of Nafion (C 1s, $292.0 \mathrm{eV}$ ). Hydrated samples (kept in distilled water) were evacuated for several hours in high vacuum (pressure lower than $5.0 \times 10^{-6} \mathrm{~Pa}$ ). PHI ACCESS ESCA-V6.0 $\mathrm{F}$ software package was used for acquisition and data analysis. A Shirley-type background was subtracted from the signals. Recorded spectra were fitted using Gauss-Lorentz curves following the methodology described elsewhere. ${ }^{1}$ Atomic concentration percentages of the elements in the membranes surfaces were determined considering the area sensitivity factor for the different measured spectral regions. ${ }^{2}$ Controlled erosion of the Nafion-117/Ag-NP membrane surface by argon sputtering ( $4 \mathrm{kV}$ and 1.5 $\mathrm{mA}$ ) during 2 minutes was also performed in order to eliminate contributions associated to surface species.

\section{SI.2. XRD measurements detailed protocol.}

XRD patterns were collected on a PANanalytical X'Pert Pro automated diffractometer. Powder patterns were recorded in Bragg-Brentano reflection configuration by using a Ge(111) primary monochromator

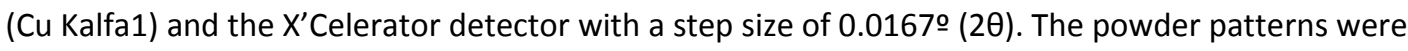
recorded between 10 and $80 \circ$ in $2 \theta$ with an equivalent counting time of ca. $60 \mathrm{sec} / \mathrm{step}$.

\section{SI.3. XANES measurements detailed protocol.}

The X-ray radiation was monochromatized by using a double crystal Si311 monochromator cooled by liquid nitrogen. The contribution of higher harmonics was rejected by a collimator and a focusing mirror by selecting on both the rhodium coating using a rejection angle of $2.0 \mathrm{mrad}$ and $0.72 \mathrm{mrad}$, respectively. This setting corresponds to a beam size of $200 \times 200 \mu \mathrm{m}^{2}$ at the sample position. Spectra were collected in transmission mode and incoming and out coming photon flux from and to the sample were measured by ionizations chambers filled with the appropriate gases. Data normalization and linear combination was done using Athena package. ${ }^{3}$ The linear combination range was -30 to $+150 \mathrm{eV}$ around the absorption Ag K-edge. The goodness of the fitting was evaluated by the R-factor. 


\section{References}

[1] M.J. Ariza, J. Benavente and E. Rodríguez-Castellón. The Capability of X-ray Photoelectron Spectroscopy in the Characterization of Membranes: Correlation between Surface Chemical and Transport Properties in Polymeric Membranes, in Handbook of Membranes: Properties, Performance and Applications, pp. 257-290. Nova Science Publishers, Inc., New York, 2009.

[2] J. Lukas and B. Jezek. Collection Czech. Chem. Commun., 1983, 48, 2909-2913.

[3] B. Ravel and M. Newville. J. Synchrotron Radiat., 2005, 12, 